\title{
Function of note strings in Japanese Tit alarm calls to the Common Cuckoo: a playback experiment
}

\author{
Jiangping Yu' ${ }^{1}$, Weiwei Lv², Hongwei Xu ${ }^{1}$, Nehafta Bibi ${ }^{1}$, Yangyang Yu' ${ }^{1}$, Yunlei Jiang ${ }^{1,3}$, Wei Liang ${ }^{4}$ \\ and Haitao Wang ${ }^{1,2^{*}}$
}

\begin{abstract}
Background: Birds produce alarm calls to convey information about threats. Some Passerine alarm calls consist of several note strings, but few studies have examined their function. Previous studies have shown that Japanese Tits (Parus minor) can alter the calling rate and number and combination of notes in response to predators. We previously found the combinations of note types in Japanese Tit alarm calls to be significantly different in response to the Sparrowhawk (Accipiter nisus) and Common Cuckoo (Cuculus canorus).
\end{abstract}

Methods: Through playback experiments, we tested whether the note strings in Japanese Tit alarm calls to the Common Cuckoo have different functions in conveying information. The note strings of selected alarm calls were divided into the categories of $C$ and $D$, and different calls were then constructed separately based on the two note string categories. Original alarm calls (C-D), C calls and D calls were played back to male Japanese Tits during the incubation period.

Results: Male Japanese Tits had a significantly stronger response to C calls than to C-D calls, and they showed a significantly stronger response to both $C$ and $C-D$ calls than to D calls, suggesting that Japanese Tits discriminated between the $C$ and $D$ calls.

Conclusions: Our study demonstrated that the C- and D-category note strings of Japanese Tit alarm calls to the Common Cuckoo have different functions, which supports the previous finding that different note strings in an alarm call can provide different information to receivers. However, the exact meanings of these note strings are not yet known, and further investigation is therefore required.

Keywords: Alarm call, Male Japanese Tit, Note strings, Function, Brood parasitism, Playback

\section{Background}

When in danger, birds usually make alarm calls (Caro 2005). Predation is perhaps the most important threat to prey, as it may lead to death or mortal injury (Lima 2009). Therefore, predation pressure is one of the forces that may drive the evolution of alarm calls (Gill and Bierema 2013; Wheatcroft and Price 2015). The alarm calls of birds to different predators may vary depending on the

\footnotetext{
*Correspondence: wanght402@nenu.edu.cn

1 Jilin Provincial Engineering Laboratory of Avian Ecology

and Conservation Genetics, School of Life Sciences, Northeast Normal

University, Changchun 130024, China

Full list of author information is available at the end of the article
}

context, such as predator type (aerial or terrestrial) and size or the predation threat level (Templeton et al. 2005; Griesser 2008; Soard and Ritchison 2009; Suzuki 2012).

Birds can benefit from the alarm calls of both conspecifics and heterospecifics that belong to the same community and share the same predators (e.g., Templeton and Greene 2007; Hetrick and Sieving 2011), providing receivers with an opportunity to escape. For example, incubating female Japanese Tits (Parus minor) responded to the alarm calls of conspecifics to snake nest predators by leaving their nests (Suzuki 2015). Alternatively, callers could recruit peers to help drive a predator away (Wheatcroft and Price 2013); for example, multiple prey 
species can combine to harass (scold, approach, or attack) perched or terrestrial predators (Hurd 1996; Langham et al. 2006). The encoding mechanisms of bird alarm calls have received much attention recently. Researchers have found that call types (Templeton et al. 2005; Suzuki 2015; Suzuki and Ueda 2013), note type combinations (Freeberg 2008; Suzuki 2014), calling rates (Wilson and Mennill 2011), the number of notes per call and the lowest frequency in a call (Leavesley and Magrath 2005), and the compositional syntax of an alarm call (Suzuki et al. 2016) can be used by birds to convey threat information to receivers.

In addition to predation pressure, brood-parasitic pressure is also an evolutionary driving force (Wheatcroft and Price 2015). Researchers classify birds that are frequently parasitized as common hosts (e.g., Yang et al. 2012; Wheatcroft and Price 2015), while birds that are rarely or never parasitized are rare hosts or non-hosts (Langmore et al. 2005). Brood parasitism increases the cost of breeding of hosts, especially common hosts (Canestrari et al. 2009); thus, common hosts develop multiple anti-parasitic strategies, such as rejecting eggs (e.g., Liang and Møller 2015; Møller et al. 2015). Many common host species emit special alarm calls when encountering brood parasites (Gill and Sealy 2004; Welbergen and Davies 2008; Wheatcroft and Price 2015; Yu et al. 2016). For example, Reed Warblers (Acrocephalus scirpaceus) repeat mobbing calls at different rates to recruit conspecifics and neighbours to chase away brood parasites (Welbergen and Davies 2008), and Yu et al. (2016) suggested that the alarm calls of Barn Swallows (Hirundo rustica) could transmit different levels of threat from the Common Cuckoo (Cuculus canorus) and the Sparrowhawk (Accipiter nisus).

Previous studies have suggested that only common hosts, and not rare hosts or non-hosts, have evolved antiparasite strategies under pressure from brood parasitism (e.g., Rothstein and Robinson 1998; Davies and Welbergen 2008; Davies 2011). Most hole-nesting bird species have been classified as "unsuitable" hosts for cuckoo parasitism because cuckoos cannot enter a cavity if the entrance is too small (van Balen et al. 1982; Moksnes et al. 1991; Davies 2000; Grim et al. 2009). However, in China, Liang et al. (2016) proved that the hole-nesting Great Tit (Parus major), a species predicted to be an unsuitable host (e.g., Moksnes et al. 1991), can reject alien eggs, indicating that hole-nesting birds may also evolve resistance mechanisms against brood parasitism (Grim et al. 2014; Liang et al. 2016).

Japanese Tits produce alarm calls in response to predators, as reported by Suzuki (2012), as well as in response to the Common Cuckoo (Yu et al. 2017). Furthermore, Suzuki (2014) demonstrated that Japanese Tits could alter the calling rate, note number and note combinations in alarm calls in response to martens and crows; for example, they produced more D notes for martens than for crows. When Japanese Tits face avian predators, $\mathrm{D}$ notes can be produced alone or in combination with other notes that appear as note strings at the end of a call (Suzuki 2014). Yu et al. (2017) also found that portions of the alarm calls of Japanese Tits in response to the Common Cuckoo contained D-note strings, and the tits even produced more $\mathrm{D}$-type notes and fewer I-type notes per call when responding to Sparrowhawks than to cuckoos.

Disentangling how the structure of bird alarm calls conveys information in different contexts is crucial for understanding the evolution of complex communicative signals. However, few studies have tested the functions of the note strings in alarm calls. Suzuki et al. (2016) demonstrated that Japanese Tits could extract different meanings from different notes and a compound meaning from different note combinations of alarm calls uttered in response to a predator. In this study, we used playback experiments to examine whether the note strings of Japanese Tit alarm calls to the Common Cuckoo have different functions in conveying information.

\section{Methods}

\section{Study area}

Our study area is located in the Zuojia Nature Reserve $\left(44^{\circ} 1^{\prime}-45^{\circ} 0 \mathrm{~N}, 126^{\circ} 0^{\prime}-126^{\circ} 8 \mathrm{E}\right)$ in Jilin Province, China. We monitored a banded population of Japanese Tits nesting in nest boxes during the breeding season (April-June) since 2011. Approximately 450 nest boxes were attached to trees approximately $3 \mathrm{~m}$ above the ground, and we visited the nest boxes at least once a week to determine the date of the first egg and clutch size (You et al. 2009).

\section{Playback experiments}

From 1 May to 13 June 2015, we conducted playback experiments with Japanese Tits during their incubation period. The alarm calls used for the playback were previously recorded from Japanese Tits exposed to a dummy Common Cuckoo above the nest boxes during the 2013 incubation period. A TASCAM HD-P2 portable digital recorder (TEAC Corporation, Tokyo, Japan) and a Sennheiser MKH P48 external directional microphone (Sennheiser electronic GmbH and Co. KG, Wedemark, Germany) were used for the recordings. The recording parameters were as follows: a frequency of $44.1 \mathrm{kHz}$ and an accuracy of 24 bits. When recording the alarm calls, we could not discriminate the individual males and females (Yu et al. 2017), but it seems that Japanese Tits cannot discriminate male and female alarm calls (Suzuki 2012, 2015). We then used Avisoft SASLab Pro 5.2 software (Avisoft Bioacoustics, Glienicke, Germany) to select 
three alarm calls from three individuals without considering their sex, and we selected one call with a high signal-to-noise ratio and no interference from heterospecific sounds or background noise from each individual. Lowfrequency $(<1 \mathrm{kHz})$ noise was filtered out. The selected alarm calls consisted of two note strings, a D-note string (a string of 7-9 D notes) and a C-note string (a combination of 2-3 C notes, Fig. 1). Then, we repeated the $\mathrm{C}$ - and $\mathrm{D}$-note strings and the original call (hereafter, the $\mathrm{C}-\mathrm{D}$ call) at a rate of 23 calls/min (the original observed calling rate) to fill a 2-min sound file for each and inserted background noise between successive calls. During the playback experiments, similar response behaviours were induced in the Japanese Tits by the same note-string categories, suggesting that one call per individual did not influence the results. Using a computer, the calls were amplified to the same level and saved in WAV format. Furthermore, we ensured that the constructed calls could be naturally pronounced alone. For the playback, $\mathrm{C}-\mathrm{D}, \mathrm{C}$ and $\mathrm{D}$ calls were used, and a background noise file was used as a control stimulus, which was created in the same way as the call files.

Playback experiments were carried out from 08:00 a.m. to 05:00 p.m. under clear and windless weather conditions. Before each treatment, we ensured that the female Japanese Tit was inside her nest box by direct observation. One set of experiments (four stimuli) for a given

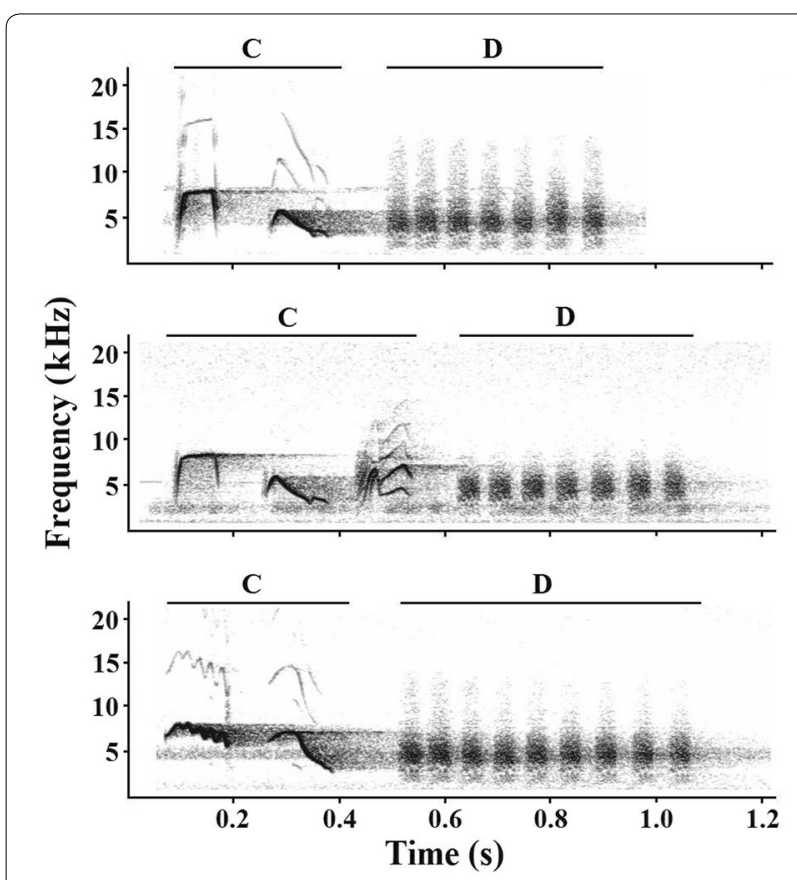

Fig. 1 Spectrographic illustration of three common C-D alarm calls (C-D refers to the original alarm calls). Blackman window, FFT-512, frame-100\%, overlap - $87.5 \%$ (bandwidth $-138 \mathrm{~Hz}$, resolution $-86 \mathrm{~Hz}$ ); noise $<1 \mathrm{kHz}$ was removed male Japanese Tit was completed in 1 day with at least a 1-h interval between each trial, and the order of the trials was counter-balanced. For each focal individual, we played back the three call types, which originated from the same calling individual to ensure that the acoustic features were constant. Each alarm call stimulus was played at the same volume, and the sound pressure level was maintained at $1 \mathrm{~m} \approx 85 \mathrm{~dB}$ with slightly larger amplitudes than the original calls (natural amplitude: $65-85 \mathrm{~dB}$ ). Thus, we could clearly discriminate the calls that came from the playback equipment from those of the focal male. If two nests were tested during the same day, the distance between the two tested nests was at least $500 \mathrm{~m}$ to avoid the response at one nest from affecting the other. For the playback, a speaker (Royqueen M300, Shenzhen, China) and a tripod were placed $5 \mathrm{~m}$ in front of the nest box, and the researchers quietly stayed $15 \mathrm{~m}$ from the nest box. To avoid subjecting focal birds to the playback sounds that originated from their own calls, we captured and identified the male Japanese Tits during the nestling period, and no adult individuals that had been banded in 2013 were recaptured. During the playback experiments, only one female Japanese Tit flew out of her nest box, and we excluded this trial from the analysis.

All behavioural responses were recorded by one person (J.Y.) to avoid variation among observers. During the playback experiments, the observer (J.Y.) was blind to the playback order since it was determined by another experimenter (W. Lv); although the observer could hear the playback, she could not discriminate the sound categories and focused on searching for and observing the focal birds. In addition, the criteria for the behavioural parameter in our observations reduced observer bias. Each experiment lasted for $12 \mathrm{~min}$, which included a 5 -min resting period to reduce the effects of human disturbance, 2 min of playback, and 5 min of observation. The behavioural responses of the male Japanese Tits were recorded beginning immediately after the stimulus was played back (Yu et al. 2016; distance from the speaker in metres and response time in seconds). We began the experiment once the male Japanese Tit could not be observed during the 5 -min rest period, and the response parameters included (1) response or not (response was the presence of the male Japanese Tit within $10 \mathrm{~m}$ of the playback speaker), (2) response latency (duration from the onset of the experiment to the response), (3) response length (duration from the onset of behavioural response to its termination, i.e., the male Japanese Tit ceased to give alarm calls and flew away, was foraging or preening or the treatment ended), (4) 2 min of parallel watching behaviour (the male Japanese Tit turned its head from left to right during the 2-min playback period), (5) 5 min of parallel watching (watching behaviour during 
the subsequent 5-min observation period; Suzuki 2012; Suzuki et al. 2016), (6) response by song, and (7) response by alarm call. Video recorders were set up to record the experimental process. The behavioural variables (1) and (4)-(7) were recorded as binary responses (yes or no).

\section{Statistical analyses}

All statistical tests were carried out in R 3.3.2 (http:// www.r-project.org) using the "stats", "psych" and "lme4" packages. Because we recorded seven behavioural variables in our study, it would have been confusing to have compared them to each other. In addition, we did not observe any special response behaviours, such as approaching the speaker, during the playback experiments. Thus, we conducted principal component analyses (PCA) to analyse the response variables. The two principal components, $\mathrm{PC} 1$ and $\mathrm{PC} 2$, complied with the Kaiser criterion (eigenvalues of 4.18 and 1.01, respectively), and the first two components explained $74 \%$ of the total variance (Table 1). Because the PC scores did not follow a normal distribution and included negative values, we transformed the data to approximately a Gamma distribution and used the transformed factor scores as response variables in the subsequent analyses. However, the interpretation of the data and the figures presented here are based on the original, untransformed PC scores. We analysed the two PC scores as dependent variables using generalized linear mixed models (GLMMs, glmer in R package lme4) with a Gamma error distribution and a log-link function. Treatment was treated as a fixed term, and the individual identity of focal birds and the playback order were treated as random effects. Since we only used three "chicka" calls from three individuals for the experimental stimuli, we could not completely avoid pseudo-replication; therefore, the identity of the playback sounds was also treated as random term. The significance of the random effects was based on likelihood ratio tests (LRT), and the level of significance was 0.10 because using LRT for this comparison is known to be conservative (Pinheiro and Bates 2000; Bates 2010). When the result of multiple comparisons was significant, we conducted post hoc tests (GLMMs). The Bonferroni correction was used to adjust the significance level because two-group comparisons after multiple comparisons increase the probability of type I errors (Rice 1989).

\section{Results}

The PC1 response scores of males to the playbacks varied significantly across the four playback stimuli (GLMMs, $\chi_{3}^{2}=156.59, n=23, p<0.001$, Fig. 2), but no significant differences in PC2 scores were detected among playback trials (GLMMs, $\chi_{3}^{2}=1.434, n=23, p=0.698$ ). PC1 explained $60 \%$ of the total variance and correlated
Table 1 Eigen values, explained variances and loadings of the behavioural measures on the two factors of the PCA

\begin{tabular}{lll}
\hline & PC1 & PC2 \\
\hline Eigenvalue & 4.18 & 1.01 \\
Variance (\%) & 60 & 14 \\
Male response or not & 0.91 & -0.24 \\
Response latency & -0.93 & 0.16 \\
Response length & 0.91 & -0.08 \\
2-Min parallel watching & 0.60 & 0.66 \\
5-Min parallel watching & 0.62 & 0.61 \\
Response by song & 0.72 & -0.23 \\
Response by alarm call & 0.62 & -0.26 \\
\hline
\end{tabular}

Loadings in italics are $>0.50$ or $<-0.50$

strongly with all behavioural variables (Table 1). According to the loading coefficients, high scores on PC1 represent strong responses (shorter response latency, longer response duration, more 2 - and 5-min horizontal scans), and the $\mathrm{PC} 1$ scores for the responses to the $\mathrm{C}$ calls were significantly stronger than for the responses to the $\mathrm{C}-\mathrm{D}$ calls (GLMMs, $n=23, p<0.001$ ). Both the PC1 scores for the responses to the $\mathrm{C}$ and $\mathrm{C}-\mathrm{D}$ calls were significantly stronger than for the responses to the D calls (GLMMs, $n=23, p<0.001$ for $C$ vs. D calls, and $p<0.001$ for $C-D$ vs. D calls). Differences in PC1 between the control (noise) and other trials were significant $(p<0.001$ for all tests).

The tests of the random effects indicated significant effects of focal individuals (LRT: $\chi^{2}=14.886, p=0.0001$ ) and non-significant effects of the order of the playbacks (LRT: $\chi^{2}=0.874, p=0.350$ ) or the identity of the playback sounds (LRT: $\chi^{2}=0, p=1.000$ ) on the response scores.

\section{Discussion}

Male Japanese Tits responded differently to playbacks of C-D, C and D calls. Suzuki et al. (2016) showed that Japanese Tits could extract a compound meaning from "ABC-D" calls uttered in response to a predator as well as different meanings from "ABC" and " $D$ " notes; "ABC" calls could elicit predator-scanning behaviour, whereas "D" calls could attract conspecifics to the callers (Suzuki et al. 2016). Although we did not observe such special response behaviours during our playback experiments, we found that male Japanese Tits exhibited a mediumstrength response to $\mathrm{C}-\mathrm{D}$ calls, a high-strength response to $\mathrm{C}$ calls and a low-strength response to $\mathrm{D}$ calls. This indicated that the $\mathrm{C}$ (similar to the "ABC" notes in Suzuki et al. 2016) and $D$ (similar to the " $D$ " notes) note strings of a C-D (similar to the "ABC-D" notes) alarm call have different functions in conveying information. Male 
Japanese Tits showed almost no response to background noise (the lowest PC1 scores, Fig. 2), but they responded to the three stimulus alarm calls, indicating that alarm calls could convey information to conspecifics.

According to our results, males obviously received some information about invaders from $\mathrm{C}$ and $\mathrm{C}-\mathrm{D}$ calls, as their responses, such as shorter response latency and longer response length, were significantly stronger than to D calls. In the Japanese Tit, females incubate eggs, and incubating females usually use conspecific alarm calls to assess the dangers outside the nest cavity (Suzuki 2015). Males do not directly participate in warming eggs, but they do play an important role in defending territories, feeding females and alarm calling to incubating females (van Duyse et al. 2002; Matysioková and Remeš 2010; Suzuki 2015). A previous study showed that incubating female Japanese Tits left their nests upon hearing conspecific "jar" calls and responded to conspecific "chicka" calls by looking from, but rarely jumping out of, their nests (Suzuki 2015). In our study, most of the females did not leave their nests $(23 / 24)$ during the playback experiments, which suggests that the alarm calls in response to the Common Cuckoo did not transmit information that an invader would enter the nests. Many males (10/23) showed parallel watching behaviour in response to $C$ calls, and a few males (4/23) showed the same behaviour in response to $\mathrm{D}$ calls during the 2-min playback period. Considering that "ABC" notes serve as warning calls (Suzuki et al. 2016), we suggest that the C-note strings of alarm calls function to transmit information about potential threats in the environment.

Japanese Tits produce " $D$ " calls to recruit conspecifics in both predatory and non-predatory contexts, such

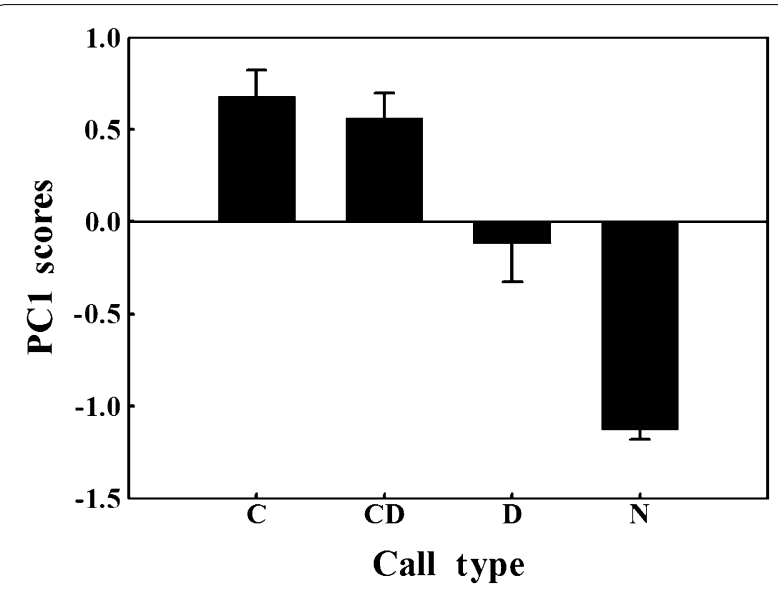

Fig. 2 PC1 scores for the responses to playbacks of $C, C-D$, and $D$ calls and background noise (control) (C-D refers to the original alarm calls, and $\mathrm{N}$ refers to the background noise; the values are presented as the mean $\pm \mathrm{SE}$ ) as when recruiting their mate to the nest (Suzuki et al. 2016). However, when we played back the D calls, the male Japanese Tits returned to the nest area but generally only quietly perched on one branch without exhibiting any special behaviours, which differed from the result of Suzuki et al. (2016). Birds can discriminate alarm call information about predators from that about nonpredators (Yu et al. 2016). For example, the D notes in the alarm calls of Black-capped Chickadees (Parus atricapillus) may convey the threat level posed by invaders (Templeton et al. 2005). As a non-predator, the Common Cuckoo should represent a lower threat level than predators, especially to hole-nesting birds, because cuckoos cannot enter cavities (van Balen et al. 1982; Moksnes et al. 1991; Davies 2000; Grim et al. 2009). Based on the results of our study, we are unsure whether Japanese Tits regard the Common Cuckoo as a brood parasite, but the D-note strings of the alarm call uttered in response to the Common Cuckoo transmits different information from the " $\mathrm{D}$ " calls in response to predators.

\section{Conclusions}

In general, the $\mathrm{C}$ - and $\mathrm{D}$-note strings of the alarm calls uttered in response to the Common Cuckoo have different functions in transmitting information to receivers. Our results support the previous finding that the different note strings of an alarm call provide different information to receivers (Suzuki et al. 2016). The C-note strings may convey information about threats around the receivers, but we could not determine the function of the D-note strings, which requires further investigation. We speculate that the information transmitted by the D-note strings varies according to the context. Further work in different contexts may help us to understand the function of variations in calling behaviour and to unravel the evolution of communication in passerines.

\section{Authors' contributions}

HW and W. Liang conceived and designed the study. JY, W. LV and HX conducted the experiments in the field. JY and HW analysed the data and wrote the paper. NB, YY, YJ and W. Liang proofread and made comments to the manuscript. All authors read and approved the final manuscript.

\section{Author details \\ ${ }_{1}^{1} \mathrm{Jilin}$ Provincial Engineering Laboratory of Avian Ecology and Conservation Genetics, School of Life Sciences, Northeast Normal University, Chang- chun 130024, China. ${ }^{2}$ Jilin Key Laboratory of Animal Resource Conservation and Utilization, Northeast Normal University, Changchun 130024, China. ${ }^{3}$ Ani- mal Science and Technology College, Jilin Agricultural University, Xincheng Street 2888, Changchun 130118, China. ${ }^{4}$ Ministry of Education Key Laboratory for Ecology of Tropical Islands, College of Life Sciences, Hainan Normal Univer-} sity, Haikou 571158, China.

\section{Acknowledgements}

We are grateful to Qianxi Fan, Dianzheng Zhao, Zhenni Liu, Siyu Zhang, Songhao Li, Yifu Sun for their assistance in fieldwork. We also thank the Zuojia Nature Reserve for the support and permission to carry out this study. This work is supported by the National Natural Science Foundation of China (31272331 and 31470458 to HW, 31472013 and 31772453 to WL), the 
Fundamental Research Funds for the Central Universities (2412016KJ043) and the Open Project Program of Jilin Provincial Key Laboratory of Animal Resource Conservation and Utilization (130028685).

\section{Competing interests}

The authors declare that they have no competing interests.

\section{Ethical standards}

The experiments comply with the current laws of China, where they were performed. Fieldwork was carried out under the permission from the Zuojia Nature Reserve, Jilin, China. Experimental procedures were permitted by National Animal Research Authority in Northeast Normal University (approval number: NENU-20080416) and the Forestry Bureau of Jilin Province of China (approval number: [2006]178).

Received: 19 December 2016 Accepted: 16 August 2017

Published online: 30 August 2017

\section{References}

Bates D. Ime4: Mixed-effects modelling with R. 2010. http://Ime4.r-forge.rproject.org/book/.

Canestrari D, Marcos JM, Baglione V. Cooperative breeding in carrion crows reduces the rate of brood parasitism by great spotted cuckoos. Anim Behav. 2009;77:1337-44.

Caro T. Antipredator defences in birds and mammals. Chicago: University of Chicago Press; 2005.

Davies NB. Cuckoos, cowbirds and other cheats. London:T and A D Poyser; 2000.

Davies NB. Cuckoo adaptations: trickery and tuning. J Zool. 2011;284:1-14.

Davies NB, Welbergen JA. Cuckoo-hawk mimicry? An experimental test. Proc R Soc Lond B. 2008;275:1817-22.

Freeberg TM. Complexity in the chick-a-dee call of Carolina chickadees (Poecile (arolinensis): associations of context and signaler behavior to call structure. Auk. 2008;125:896-907.

Gill SA, Bierema AMK. On the meaning of alarm calls: a review of functional reference in avian alarm calling. Ethology. 2013;119:449-61.

Gill SA, Sealy SG. Functional reference in an alarm signal given during nest defence: seet calls of yellow warblers denote brood-parasitic brownheaded cowbirds. Behav Ecol Sociobiol. 2004;56:71-80.

Griesser M. Referential calls signal predator behavior in a group-living bird species. Curr Biol. 2008;18:69-73.

Grim T, Rutila J, Cassey P, Hauber ME. The cost of virulence: an experimental study of egg eviction by brood parasitic chicks. Behav Ecol. 2009;20:1138-46.

Grim T, Samaš P, Procházka P, Rutila J. Are tits really unsuitable hosts for the common cuckoo? Ornis Fennica. 2014:91:166-77.

Hetrick SA, Sieving KE. Antipredator calls of tufted titmice and interspecific transfer of encoded threat information. Behav Ecol. 2011;23:83-92.

Hurd CR. Interspecific attraction to the mobbing calls of black capped chickadees (Parus atricapillus). Behav Ecol Sociobiol. 1996;38:287-92.

Langham GM, Contreras TA, Sieving KE. Why pishing works: Titmouse (Paridae) scolds elicit a generalized response in bird communities. Ecoscience. 2006;13:485-96.

Langmore NE, Kilner RM, Butchart SHM, Maurer G, Davies NB, Cockburn A, Macgregor NA, Peters A, Magrath MJL, Dowling DK. The evolution of egg rejection by cuckoo hosts in Australia and Europe. Behav Ecol. 2005;16:686-92.

Leavesley AJ, Magrath RD. Communicating about danger: urgency alarm calling in a bird. Anim Behav. 2005;70:365-73.

Liang W, Møller AP. Hawk mimicry in cuckoos and anti-parasitic aggressive behavior of barn swallows in Denmark and China. J Avian Biol. 2015;46:216-23.

Liang W, Møller AP, Stokke BG, Yang C, Kovařík P, Wang H, Yao C-T, Ding P, Lu X, Moksnes A, Røskaft E, Grim T. Geographic variation in egg ejection rate by great tits across 2 continents. Behav Ecol. 2016;27:1405-12.
Lima SL. Predators and the breeding bird: behavioral and reproductive flexibility under the risk of predation. Biol Rev. 2009;84:485-513.

Matysioková B, Remeš V. Incubation feeding and nest attentiveness in a socially monogamous songbird: role of feather colouration, territory quality and ambient environment. Ethology. 2010;116:596-607.

Moksnes A, Røskaft E, Braa AT, Korsnes L, Lampe HM, Pedersen HC. Behavioural responses of potential hosts towards artificial cuckoo eggs and dummies. Behaviour. 1991;116:64-89.

Møller AP, Stokke BG, Samia D. Hawk models, hawk mimics and anti-predator behavior of prey. Behav Ecol. 2015;26:1039-44.

Pinheiro JC, Bates D. Mixed effects models in S and S-Plus. New York: Springer; 2000.

Rice WR. Analyzing tables of statistical tests. Evolution. 1989;43:223-5.

Rothstein SI, Robinson SK. Parasitic birds and their hosts: studies in coevolution. Oxford: Oxford University Press; 1998.

Soard CM, Ritchison G. "Chick-a-dee" calls of Carolina chickadees convey information about degree of threat posed by avian predators. Anim Behav. 2009:78:1447-53.

Suzuki TN. Referential mobbing calls elicit different predator-searching behaviours in Japanese great tits. Anim Behav. 2012:84:53-7.

Suzuki TN. Communication about predator type by a bird using discrete, graded and combinatorial variation in alarm calls. Anim Behav. 2014;87:59-65.

Suzuki TN. Assessment of predation risk through referential communication in incubating birds. Sci Rep. 2015;5:10239.

Suzuki TN, Ueda K. Mobbing calls of Japanese tits signal predator type: field observations of natural predator encounters. Wilson J Ornithol. 2013;125:412-5.

Suzuki TN, Wheatcroft D, Griesser M. Experimental evidence for compositional syntax in bird calls. Nat Commun. 2016;7:10986.

Templeton CN, Greene E. Nuthatches eavesdrop on variations in heterospecific chickadee mobbing alarm calls. Proc Natl Acad Sci USA. 2007:104:5479-82.

Templeton CN, Greene E, Davis K. Allometry of alarm calls: black-capped chickadees encode information about predator size. Science. 2005;308:1934-8.

van Balen JH, Booy CJH, Van Franeker JA, Osieck ER. Studies on hole-nesting birds in natural nest sites. 1. Availability and occupation of natural nest sites. Ardea. 1982;70:1-24.

van Duyse E, Pinxten R, Eens M. Effects of testosterone on song, aggression, and nestling feeding behavior in male great tits, Parus major. Horm Behav. 2002;41:178-86.

Welbergen JA, Davies NB. Reed warblers discriminate cuckoos from sparrowhawks with graded alarm signals that attract males and neighbours. Anim Behav. 2008;76:811-22.

Wheatcroft D, Price TD. Learning and signal copying facilitate communication among bird species. Proc R Soc B Biol Sci. 2013;280:20123070.

Wheatcroft D, Price TD. Rates of signal evolution are associated with the nature of interspecific communication. Behav Ecol. 2015;26:83-90.

Wilson DR, Mennill DJ. Duty cycle, not signal structure, explains conspecific and heterospecific responses to the calls of black-capped chickadees (Poecile atricapillus). Behav Ecol. 2011;22:784-90.

Yang C, Liang W, Antonov A, Cai Y, Stokke BG, Fossøy F, Moksnes A, Røskaft E. Diversity of parasitic cuckoos and their hosts in China. Chin Birds. 2012;3:9-32.

You Y, Feng J, Wang H, Wang J, Dong C, Su X, Sun H, Gao W. Variation in egg size and nestling growth rate in relation to clutch size and laying sequence in great tits Parus major. Prog Nat Sci. 2009;19:427-33.

Yu J, Wang L, Xing X, Yang C, Ma J, Møller AP, Wang H, Liang W. Barn swallows (Hirundo rustica) differentiate between common cuckoo and sparrowhawk in China: alarm calls convey information on threat. Behav Ecol Sociobiol. 2016;70:171-8.

Yu J, Xing X, Jiang Y, Liang W, Wang H, Møller AP. Alarm call-based discrimination between common cuckoo and Eurasian sparrowhawk in a Chinese population of great tits. Ethology. 2017;123:542-50. 